\title{
Adult Orthodontics Versus Adolescent Orthodontics: An Overview
}

\author{
Dinesh K. Bagga
}

\section{ABSTRACT}

The scope of orthodontics has widened to include not only children and adolescents but also adults, thereby abolishing the upper age limit. With growing esthetic conscience among the society, a great number of young adults are seeking orthodontic treatment primarily for esthetic reasons. They undergo comprehensive orthodontic treatment involving major occlusal changes to get the utmost esthetically pleasing face. The older adults with poor dental conditions requiring perio-restorative treatment undergo adjunctive orthodontic treatment to attain a long-term prognosis. The orthodontist faces challenges to practice adult orthodontics due to various issues being under considerations, which are quite different than routine orthodontic treatment oriented to children and adolescents. This article highlights the difficulties and limitations faced by the orthodontist while practicing adult orthodontics with remedies to overcome them.

\section{KEY WORDS}

Adult Orthodontics. 


\section{INTRODUCTION}

here is a rising influx of adult patients seeking orthodontic treatment in the recent era. 20-25\% of orthodontic patients are reported to be adults and this trend is likely to rise by leaps and bounds in the near future in view of society becoming more esthetic and health conscious. Hence, it is imperative to explore and understand various aspects of orthodontic treatment where adults need special considerations in contrast to adolescents. Adult orthodontics is basically same as adolescent orthodontics for tissue changes associated with tooth movement, stages of treatment and goal of treatment. But there are certain differences in several aspects namely psychosocial, biological and mechanical aspects where adults need special consideration for behavioral and clinical management (1). Adult patients require different treatment approach from adolescents.

\section{ISSUES TO BE CONSIDERED}

Various factors must be given considerations, which demand special consideration for adults.

- Psychosocial factors

- Perio-restorative problems

- Age related considerations

- Lack of growth potential

- Aging of tissues

- Vulnerability to Root resorption

- Vulnerability to TMD

To take care of these important issues, adult orthodontics often requires interdisciplinary approach to deliver efficient treatment outcome involving many healthcare providers viz. Periodontist, Restorative Dentist, Prosthodontist, Endodontist, TMJ specialist, Oral \& Maxillofacial Surgeon etc (2).

\section{Psychosocial factors}

It is important to understand expectations and attitude of adult patients in order to plan behavioral management before clinical management. Adult patients have high treatment expectations. They are more inquisitive about the detail of the treatment as treatment time, complexity of treatment, number of visits, likelihood of correction etc. They have been shown to have more discomfort from appliances. They are more co-operative in following the instructions from orthodontists such as elastic wear, hygiene maintenance, keeping their appointments etc but they don't commit to long-term treatment (3). In other words, adults demand best treatment results in a short time. Therefore, it is quite important to apprise these patients about the limitations \& complexity of the treatment, increased treatment time \& high relapse potential.

Adult patients may have hesitation in accepting visibility of orthodontic appliances. They may demand esthetic appliance e.g. esthetic brackets, lingual appliance, invisalign etc irrespective of their limitations (3).

\section{Perio-restorative problems and age related} issues

These issues need special considerations in adult orthodontics.

\section{GOAL OF ORTHODONTIC TREATMENT}

Though acceptable esthetics is an integral part of treatment goal at any age, but function, stability and health of dentition are given paramount importance in adults. Adult patients have many preexisting conditions that are usually not present in adolescent patients. Hence, additional treatment objectives are established at the outset of the treatment $(1,4)$.

Additional treatment objectives are determined to facilitate and improve effectiveness of periorestorative treatment by:

- Improving axial inclination of teeth, (to have sufficient bone between roots for good vascular supply and proper contact area);

- Achieving parallelism of abutment teeth (to minimize tooth cutting, axial loading \& properly sized replacement for fabrication of prosthesis);

- Most favorable distribution of abutment teeth (to receive prosthesis for better stability);

- Uprighting and extrusion of posterior teeth with occlusal equilibration sometimes followed by endodontic treatment (to improve vertical osseous defects, axial loading and crown-root ratio);

- Forced extrusion of those teeth which get damaged upto one third of cervical line (to improve accessibility, 
increase biological width and provide better support at the margin of the prosthesis);

- To restore functional occlusion keeping in mind existing skeletal relationship rather than aiming for Andrew's six keys to normal occlusion;

- Achieving better lip support for flaccid \& long upper lip by maintaining anterior teeth in slight procumbent position with correction of overjet by proclining \& maintaining lower incisors in more procumbent position than normal position to avert wrinkles around the lips

- Restoring vertical dimension with bite plate before placing prosthesis in bite collapse and tooth mobility.

\section{TREATMENT MODALITIES AVAILABLE}

Correction of skeletal anomaly involves either surgery or dental camouflage (extraction of tooth to allow rest of teeth getting accommodated and masking abnormal jaw relationship). Growth modification appliances are not opted for adults due to completion of growth in contrast to adolescents (5).

\section{CONSIDERATIONS REGARDING EXTRACTION}

Extraction choice may be affected by periorestorative status of dentition or already extracted tooth complicating the treatment plan. Space closure may be difficult especially in molar region.

Old extraction site common in adults poses mechanical and biological challenges. Maintenance of closed spaces is very unpredictable (difficult to close and keep it closed) (6). Response of cortical bone to orthodontic force is significantly slow. It may need uprighting to open the space mesially to receive prosthesis rather than attempting space closure.

Uprighting of molars has several advantages:

Correction of vertical osseous defect on mesial side

- Elimination of gingival folding and plaque retentive area on the mesial side

- Parallellism of abutment (less tooth cutting required and axial loading of prosthesis)

- Proper sized prosthesis

For extraction site created by loss of tooth due to periodontal disease, it is better to move teeth away to restore it with prosthesis since normal bone formation may not occur if tooth is moved into the defect (7).

Existing occlusion is maintained when occlusal difficulties are not present. Lower incisor extraction is preferred over bicuspid extraction to relieve crowding. Proximal stripping (as 3 cornered spaces are more prevalent) and occlusal equilibration are frequently done procedures (3).

\section{CONSIDERATIONS REGARDING APPLIANCE PLACEMENT}

While bonding, special considerations may be required due to presence of restorations such as porcelain and metallic surfaces (8).

All restorations must be properly polished and excess adhesive around orthodontic attachments should be removed. Strict oral hygiene procedures must be reinforced to clean various difficult areas. Stainless steel ligatures may be preferred to elastomeric modules for being less retentive to plaque and also causing less friction(9).

\section{BIOMECHANICAL CONSIDERATIONS}

Adult bone is less reactive to mechanical forces (10). There is a greater risk of loss of attachment as well as marginal bone loss with mild gingival infections as compared to adolescents (11). Marginal bone loss and gingival recession are commonly present in adults (12). Affected occlusal function may show disuse dystrophy in their supporting bone (13). Loss of attachment leads to apical shift of centre of resistance thereby increasing distance from centre of resistance to point of force application in turn leading to increased tipping moment produced by the given force. Therefore greater countervailing moment is required to balance this greater tipping moment to translate periodontally compromised tooth $(7,14)$.

Forces between active \& reactive units are balanced considering the number of teeth to be moved, anchorage available \& direction/amount of movement.

Same force produces greater pressure in periodontally compromised tooth than that in a healthy tooth due to reduced periodontal tissue. Hence, the absolute magnitude 
of force should be reduced also due to other reasons such as delayed response (due to reduced cellular activity in adults) and risk of root resorption (due to dense cortical bone and decreased periodontal width) (14-17).

\section{Considerations Regarding Tooth Movements}

To correct deep bite in young patients, posterior extrusion is allowed because of compensation made by vertical growth. But overbite correction in adults should be carried out by intrusion of anterior teeth, not by extrusion of posterior teeth $(1,5,7)$. This is because posterior extrusion would encroach in the freeway space due to lack of vertical growth stressing the TMJ muscles and result in downward and backward movement of the mandible, which thereby tend to relapse due to instability.

If individual tooth extrusion is required to level the associated vertical bone defect, the tooth should be occlusally equilibrated (4).

Palatal expansion is carefully done to avoid buccal tipping due to extrusion associated with it.

Intrusion leads to deepening of periodontal pockets in periodontally involved patients. It has been observed that in healthy patients, periodontal probing depth does not increase rather improves gingival position forming a tight epithelial cuff. Therefore intrusion should never be attempted without control of inflammation (7).

Most mechanotherapy has extrusive component. Retraction force has a larger extrusive force component if the marginal bone loss is most pronounced. Hence, light continuous intrusive force should be maintained during retraction (7).

In adult patients segmented arch mechanics (a stable anchor unit by several teeth rigidly connected to create functional equivalent of a single large multirooted anchor tooth) is preferred for intrusion. Light force is required for adults with periodontally compromised status due to less periodontal area for force distribution. Mechanotherapy for intrusion is same for adults \& adolescents.

- Burstone type depressing arches

- Rickett's utility arches
Both have long span from stabilized posterior segments to anterior area but Burstone type depressing arches are preferred in adults due to more flexibility and thereby delivering light force (7).

\section{Considerations Regarding Anchorage}

Anchorage may be affected by poor periorestorative status and missing teeth (18). Headgears may not be acceptable to an adult due to esthetic reasons. Hence intraoral anchorage devices such as posterior stabilizing segments (using lingual arches and buccal stabilizing wires) and controlled forces are used. Two step space closure with frictionless mechanics can be used to reduce strain on anchorage (frequent appointments may be needed for the control of cuspid retraction) (7). Microimplants can also be used to avoid dependence on teeth for anchorage (19).

\section{CONSIDERATIONS REGARDING VULNERABILITY TO ROOT RESORPTION}

Adult patients must be informed about the risk of root resorption and thoroughly evaluated for the susceptibility to root resorption $(1,5,10,17,20)$. All measures should be taken to manage root resorption.

Patients should be evaluated for any sign of root resorption at the outset of the treatment (family history, habits, prolonged treatment time, long and narrow root forms, $\mathrm{h} / \mathrm{o}$ trauma etc.). If no sign of root resorption exists at the start of orthodontic treatment, it is further evaluated after 6-9 months of treatment using IOPA radiographs. On discovering any sign at the outset, root resorption needs to be evaluated with radiographs every 3 months thereon. On discovering any sign mid-treatment, all forces should be withdrawn for about 8 weeks. Orthodontic treatment can be resumed after cessation of root resorption. Up to $1 / 3^{\text {rd }}$ of root length loss does not hinder with the function significantly (1). If it does not stop with the best of the efforts (use of light and intermittent forces, avoidance of jiggling forces, intrusion with special precautions), orthodontic treatment may need to be abandoned. If root resorption still continues, sequential root canal treatment should be done initially with calcium hydroxide followed by gutta-percha only after root resorption ceases (20). 


\section{CONSIDERATIONS REGARDING VULNERABILITY TO TMD}

There is a higher risk of developing TMD in adults than adolescents, which may not be related to orthodontic treatment. Hence, adult patients need a thorough check up for the signs of TMD before initiation of orthodontic treatment.

Adult patients may seek orthodontic treatment due to TMD. They need to be explained about the risk of developing TMD not necessarily related to orthodontic treatment and limitations of orthodontic treatment in the management of $\operatorname{TMD}(7,21)$.

\section{CONSIDERATIONS REGARDING TREATMENT TIME}

Tissue remodeling associated with tooth movement is slow leading to slow rate of tooth movement making the treatment time longer $(22,23)$. Activation in adults usually in 50's and onwards is required to be done after longer period i.e. 3-6 weeks as against 2-4 weeks required in adolescents. Initiation of tooth movement takes longer time as compared to adolescents. The delayed response to mechanical stimulus, is suggested to be caused by insufficient source of preosteoblasts as a result of reduced vascularization with increasing age (24). After delayed initial tissue reaction, rate of tooth movement in adults is not that much different as compared to that in adolescents. Total treatment time can be made at par or a little longer in adults if good cooperation is achieved from an adult patient, which makes up for initial slower tooth movement (25).

\section{CONSIDERATIONS REGARDING FINAL DETAILING AND RETENTION}

Final finishing is done with archwires \& then stabilized with immediately placed retainers. Positioners are less often indicated as finishing devices especially for adults with moderate to severe bone loss (7). Eventual detailing of occlusal relationships is done in adults by equilibration, tooth reshaping, incisal edge equilibration and also marginal ridge reduction as frequently done procedures $(3,7)$. Healthy adults end up with stable occlusion. High relapse tendency is reported in adults in contrast to adolescents $(1,3,5,7,10)$. So, prolonged retention is required due to reduced cellular activity along with increase in lag time for tissue remodelling in adult patients $(3,10)$. Periodontally compromised patients may need permanent retention $(26,27)$.

Traditional orthodontic retainer (to allow each tooth to move independently) is not indicated in adults with significant periodontal bone loss \& mobile teeth. Splinting may be required either short term being occlusal splint, wraparound retainer, a suckdown plastic wafer or long term using cast restorations (7).

\section{CONCLUSION}

An individualized appropriate treatment plan needs to be formulated for an adult patient on the basis of careful evaluation of a complex interaction of various biological, psychosocial and mechanical factors.

\section{REFERENCES}

1. Vanarsdall RL, Musich DR. Adult orthodontics: Diagnosis and treatment. In: Graber TM, Vanarsdall RL, Vig KWL (eds). Orthodontics: Current principles and techniques. $4^{\text {th }}$ edition, St Louis: Mosby, 2005: 937-992.

2. Kalia S, Melsen B. Interdisciplinary approaches to adult orthodontic care. J Orthod 2001; 28(3): 191-196.

3. Sheridan JJ. The reader's corner. J Clin Orthod 2005; 39(4): 219223.

4. Kokich $\mathrm{V}$. The role of orthodontics as an adjunct to periodontal therapy, In: Newman MG, Takei HH, Carranza FA, eds. Clinical periodontology, $9^{\text {th }}$ edn, Philadelphia, WB Saunders Co. 2002:704718.

5. Bishara SE: Textbook of orthodontics. WB Saunders Co. 2001: 494-531.

6. Hom BM, Turley PK. Effects of space closure of the mandibular first molar area in adults. Am J Orthod Dentofac Orthop 1984; 105 : 25-34.

7. Proffit WR, Fields HW, Sarver DM. Contemporary Orthodontics. $4^{\text {th }}$ Ed, St Louis, Mosby. 2007: 635-685.

8. Zachrisson BU, Buyukyilmaz T. Recent advances in bonding to gold, amalgam and porcelain. J Clin Orthod 1993; 27: 661-675.

9. Forsberg CM, Brattstrom V, Malmberg E, Nord CE. Ligature wires and elastomeric rings: two methods of ligation and their association with microbial colonization of Streptococcus mutans and Lactobacilli. Eur J Orthod 1991; 13: 416-420.

10. Melsen B. Limitations in adult orthodontics. In: Melsen B (ed). Current controversies in orthodontics. Quintessence Publishing Co. 1991: 147-180.

11. Boyd RL, Leggott PQ, Quinn RS, et al. Periodontal implications of orthodontic treatment in adults with reduced or normal periodontal tissues versus those of adolescents. Am J Orthod Dentofacial Orthop 1989; 96: 191-198.

12. Shei O, Waerhaug J, Lovdal A, Arnulf A. Alveolar bone loss as related to oral hygiene and age. J Periodontol 1959; 26: 7-16. 
13. Picton DCA. The effect of external forces on the periodontium. In: AH Melcher, $\mathrm{WH}$ Bowen (eds) Biology of the periodontium. New York, Academic Press, 1969: 363419

14. Williams S, Melsen B, Agerbaek N, Asboe V. The orthodontic treatment of malocclusion in patients with previous periodontal disease. Br J Orthod 1982; 9: 178-184

15. Reitan K. Effects of force magnitude and direction of tooth movement on different alveolar bone types. Angle Orthod 1964; 34(4): 244-255.

16. Reitan K. Initial tissue behavior during apical root resorption. Angle Orthod 1974; 44: 68-82.

17. Mirabella AD, Artun J. Risk factors for apical root resorption of maxillary anterior teeth in adult orthodontic patients. Am J Orthod Dentofac Orthop 1995; 108: 4855.

18. Ong MA, Wang HL, Smith FN. Interrelationships between periodontics and adult orthodontics. J Clin Periodontol 1998; 25: 271-277.

19. Bryant SR, Zarb GA. Osseointegration of oral implants in older and younger adults. Int J Oral Maxillofac Implants 1998; 13: 492-499.
20. Malmgren O, Levander E. Minimizing orthodontically induced root resorption. In Graber TM, Eliades T, Athanasiou AE (eds). Risk management in orthodontics: Experts' guide to malpractice. Quintessence Publishing Co. 2004: 6174.

21. McNamara JA, Seligman DA, Okeson JP. Occlusion, orthodontic treatment and temporomandibular disorders: a review. J Orofacial Pain 1995; 9: 73-90.

22. Bond JA. The child versus the adult. Dent Clin North Am 1972; 16: 401-412.

23. Reitan K. Tissue reaction as related to the age factor. Dental record. 1954; 74: 271 279.

24. Norton LA. The effect of aging cellular mechanisms on tooth movement. Dent Clin North Am 1988; 32: 437-446.

25. Dyer GS, Harris EF, Vaden JL. Age effects on orthodontic treatment: adolescents contrasted with adults. Am J Orthod Dentofac Orthop 1991; 100: 523-530.

26. Harris EF, Vaden JL, Dunn KL. Effects of patient age on post orthodontic stability in Class II, division 1 malocclusion. Am J Orthod Dentofac Orthop 1994; 105: 2534.

27. Kahl-Nieke B. Retention and stability considerations for adult patients. Dent Clin North Am 1996; 40: 961-994.

\section{THE AUTHOR}

\section{Dr. Dinesh K. Bagga}

Professor and Head

Deptt. of Orthodontics and Dentofacial

Orthodpaedics,

I.T.S. Centre for Dental

Studies and Research,

Murad Nagar,

Ghaziabad (UP)

Phone no. 09868071583

E mail: drdkbagga2000@yahoo.co.in 